\title{
MUON CAPTURE AND COOLING DYNAMICS, CAPTURE IN SOLENOIDAL CHANNELS*
}

\begin{abstract}
G. Penn, UC Berkeley / LBNL Center for
Abstract

Ionization cooling is a crucial component of either a muon collider or a neutrino factory based on muon decays. It determines the number of muons, per proton on target, that fall into the acceptance of the accelerator and storage ring. Current studies of cooling channels predominantly use simulations which track many particles, an often time consuming procedure. Analytic models [K.-J. Kim and C.X. Wang, Phys. Rev. Lett. 85(4):760-763; G. Penn and J.S. Wurtele, Phys. Rev. Lett. 85(4):764-767] using equations for the beam moments have been developed. These dynamic equations, similar to the Courant-Snyder description of quadrupole focussing, incorporate the basic aspects of ionization cooling: energy loss and scattering in material, acceleration by radio frequency (rf) cavities, and focussing in solenoid magnets. The moments method is compared to simulations and shown to provide for a reasonable prediction of the percentage of muons captured within a defined lattice acceptance, which is the figure of merit that is customarily used for evaluating the performance of cooling channels in simulations. The moments method is used to evaluate the impact on channel performance of engineering constraints and beam structure.
\end{abstract}

\section{INTRODUCTION}

There is strong interest in the preparation and acceleration of muon beams, for a muon collider [1] or for the production of an intense neutrino beam [2]. The primary muon source being considered is from the pions produced when a proton beam is directed onto a target. The resulting muons are expensive to produce and occupy a phase space region much too large to be used for either facility. The six-dimensional phase space volume must be reduced by a factor of order 100 before it can be useful for the neutrino factory, or $10^{6}$ for the muon collider.

The cooling must be accomplished more quickly than the muon lifetime, on the order of $2 \mu \mathrm{s}$. The cooling method proposed is ionization cooling [3], where particles are slowed down in material and then reaccelerated using radio frequency (rf) cavities. Because momentum is lost in all directions, but only added in the forward direction, the spread in transverse angles is reduced. Competing against this transverse cooling are multiple scattering events, and to achieve effective cooling the beam must be focussed to a small spot size. In addition, because the functional dependence of energy loss in material tends to increase spreads in energy, transverse cooling is associated with some lon-

\footnotetext{
* Work supported by the U.S. Department of Energy, grant DEFG-0395ER-40936.
}

gitudinal heating, leading to particle losses as they fall out of the rf bucket.

Further challenges to cooling the beam arise from engineering aspects of realistic cooling channels, which contain apertures, in particular windows in the rf cavities, that will scrape a significant portion of the uncooled beam. The large beam emittance and strong focussing also introduces significant corrections to the paraxial approximation, and nonlinear dynamic effects of beam propagation can lead to excessive particle losses if not properly taken into account.

This paper describes a set of paraxial moments equations, similar in form to the Courant-Snyder formalism [4], which incorporates the main components of ionization cooling channels: momentum loss in material, acceleration by rf, multiple scattering, and particle decays. In addition, to obtain good agreement with particle tracking codes, and to model physics and engineering considerations which apply outside the paraxial approximation. In particular, longitudinal emittance growth and beam scraping have been incorporated into the code which solves these equations, and nonlinear beam correlations have been analyzed to aid in properly matching the beam to obtain peak channel performance.

\section{MOMENTS EQUATIONS}

The moments equations are derived from the single particle equation of motion, with the inclusion of a stochastic term for the effect of multiple scatter. Here, a brief description of the equations used is provided. For a more detailed description, see Refs. [5] and [6]. It is useful to first consider single particle motion without the diffusion in angle resulting from multiple scatter, but including acceleration in rf cavities and energy loss in material.

In the paraxial approximation, the forward momentum evolves as

$$
P_{z}^{\prime} \simeq\left(q E_{z} / v_{z}\right)+(1 / v) \frac{d E}{d s},
$$

and in a quadrupole lattice the equations of motion are:

$$
\begin{aligned}
& x^{\prime \prime}+x^{\prime} \frac{q E_{z}}{v_{z} P_{z}}+K x=0, \\
& y^{\prime \prime}+y^{\prime} \frac{q E_{z}}{v_{z} P_{z}}-K y=0,
\end{aligned}
$$

where $K=q B^{\prime} / P_{z}$. Note that the energy loss in material does not explicitly appear in these equations, and that the two degrees of freedom are completely uncoupled.

In solenoid lattices, it is traditional to use a reference frame that is rotating with inverse length $\kappa=q B_{z} / 2 P_{z}$, which is half of the cyclotron rotation rate. In vacuum, the 
two transverse directions are decoupled in this frame; the full equations including the effect of material are:

$$
\begin{aligned}
x_{R}^{\prime \prime}+x_{R}^{\prime} \frac{q E_{z}}{v_{z} P_{z}}+\kappa^{2} x_{R} & =\kappa y_{R} \frac{1}{v P_{z}} \frac{d E}{d s}, \\
y_{R}^{\prime \prime}+y_{R}^{\prime} \frac{q E_{z}}{v_{z} P_{z}}+\kappa^{2} y_{R} & =-\kappa x_{R} \frac{1}{v P_{z}} \frac{d E}{d s} .
\end{aligned}
$$

The RHS couples the two transverse directions when material is present inside the magnetic field; this corresponds to a source of canonical angular momentum.

The beam is assumed cylindrically symmetric, with the second order moments parametrized by

$$
\begin{aligned}
\left\langle x^{2}\right\rangle & =\epsilon_{N} \beta_{\perp} m c / P_{z}, \\
\left\langle x P_{x}\right\rangle & =-\epsilon_{N} \alpha_{\perp} m c, \\
\left\langle L_{\text {canon }}\right\rangle & =2 m c \epsilon_{N} \mathcal{L},
\end{aligned}
$$

and the determinant of the $4 \mathrm{X} 4$ covariance matrix is equal to $\left(m c \epsilon_{N}\right)^{4}$.

The dynamic equations for these parameters are solved in the paraxial limit; in vacuum, the emittance is constant and the beta function satisfies

$$
2 \beta_{\perp} \beta_{\perp}^{\prime \prime}-\left(\beta_{\perp}^{\prime}\right)^{2}+4 \beta_{\perp}^{2} \kappa^{2}-4\left(1+\mathcal{L}^{2}\right)=0 .
$$

More generally,

$$
\begin{aligned}
\epsilon_{N}^{\prime} & =\beta_{\perp} \frac{P \mathcal{S}}{2 m c}+\epsilon_{N} \frac{1}{v_{z} P} \frac{d E}{d s} \\
\beta_{\perp}^{\prime} & =-2 \alpha_{\perp}+\beta_{\perp} \frac{q\left\langle E_{z}\right\rangle}{v_{z} P_{z}}-\frac{\beta_{\perp}^{2}}{\epsilon_{N}} \frac{P \mathcal{S}}{2 m c} \\
\alpha_{\perp}^{\prime} & =-\gamma_{\perp}+2 \kappa\left(\beta_{\perp} \kappa-\mathcal{L}\right)-\frac{\alpha_{\perp} \beta_{\perp}}{\epsilon_{N}} \frac{P \mathcal{S}}{2 m c} \\
\mathcal{L}^{\prime} & =-\beta_{\perp} \kappa \frac{1}{v_{z} P} \frac{d E}{d s}-\frac{\mathcal{L} \beta_{\perp}}{\epsilon_{N}} \frac{P \mathcal{S}}{2 m c} \\
\left\langle P_{z}\right\rangle^{\prime} & =\frac{q\left\langle E_{z}\right\rangle}{v_{z}}+\frac{1}{v} \frac{d E}{d s} .
\end{aligned}
$$

The source term $\mathcal{S}$ for spread in transverse angles due to multiple scatter is $\mathcal{S}=(13.6 \mathrm{MeV})^{2} /\left(2 m c v^{2} P L_{R}\right)$, where $L_{R}$ is the radiation length of the material.

We can also define a transverse amplitude $A$ as

$$
\begin{aligned}
A= & \frac{P_{z}}{m c}\left[\frac{x^{2}+y^{2}}{\beta_{\perp}}+\beta_{\perp}\left(x^{\prime}+\frac{\alpha_{\perp}}{\beta_{\perp}} x-\frac{\beta_{\perp} \kappa-\mathcal{L}}{\beta_{\perp}} y\right)^{2}\right. \\
& \left.+\beta_{\perp}\left(y^{\prime}+\frac{\alpha_{\perp}}{\beta_{\perp}} y+\frac{\beta_{\perp} \kappa-\mathcal{L}}{\beta_{\perp}} x\right)^{2}\right]
\end{aligned}
$$

A distribution function which depends purely on $A$ will have consistent values of $\beta_{\perp}, \alpha_{\perp}$, and $\mathcal{L}$.

\section{EXTENSIONS TO MOMENTS EQUATIONS}

The paraxial equations alone are not sufficient to accurately model cooling channels when realistic beam parameters are used. The main reasons for this are that the rf bucket is typically almost full, so that longitudinal mismatch or emittance growth leads to particle losses; beam sizes are large enough that apertures scrape significant portions of the beam; and nonlinear effects introduce additional matching constraints. The dominant nonlinear effect is the dependence of longitudinal motion on transverse motion, because corrections to transverse motion itself are of higher order. In the case where cooling rates and synchrotron motion are slow compared to betatron motion, this nonlinearity can be simplified to dynamic equations for energy and time which depend on transverse amplitude.

\subsection{LONGITUDINAL EMITTANCE GROWTH}

To first order, the longitudinal emittance growth is given by $\epsilon_{L}^{\prime} \simeq \epsilon_{L}(d / d E)(d E / d s)$. There is additional emittance growth due to straggling, but for large emittance beams, where typical values for $\epsilon_{L}$ are larger than $20 \mathrm{~mm}$, this is a small contribution. One can also define longitudinal $\alpha_{L}$ and $\beta_{L}$, in analogy with transverse parameters. However, because typical cooling channel parameters call for an rf bucket which is almost full to maximize the rate of acceleration, this is not a very useful consideration because longitudinal phase space manipulations are precluded; the beam must be kept matched to the rf at all times. The exceptions are during so-called "phase rotation" and bunching, which are not best represented by a moments formalism.

\subsection{BEAM SCRAPING}

Because of the large initial transverse emittance of the muon beam, some particles are lost against narrow apertures in the channel. As the high amplitude particles are being scraped, this does not necessary result in a severe penalty in the figure of merit of muons finally delivered into the downstream acceptance. However, for the purpose of comparisons, and to determine the limits at which apertures degrade performance, it is essential to have a reasonable model for scraping processes in a channel.

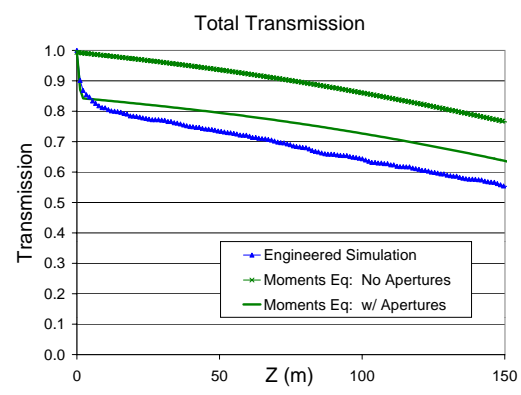

Figure 1: Transmission of particles in FOFO cooling channel. ICOOL simulation is compared with moments equations both with and without the beam scraping model.

In Figures 1 - 3, the results of the simulation of a realistic "FOFO"-type cooling channel using ICOOL [7], a particle 


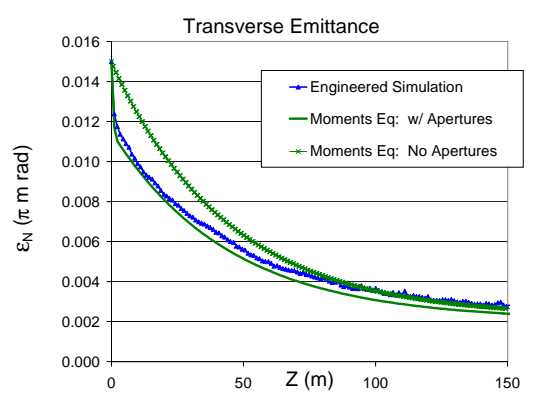

Figure 2: Transverse emittance in FOFO cooling channel. ICOOL simulation is compared with moments equations both with and without the beam scraping model.

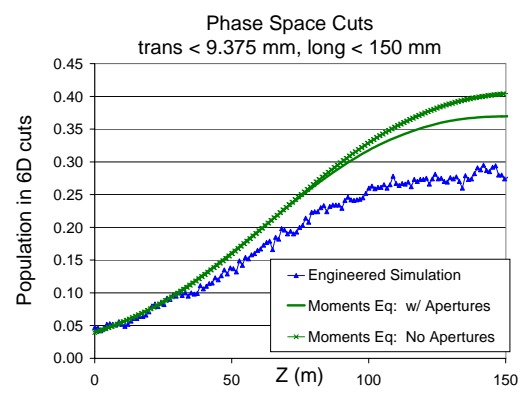

Figure 3: Evolution of figure of merit, number of particles in downstream phase space acceptance region, in FOFO cooling channel. ICOOL simulation is compared with moments equations both with and without the beam scraping model.

tracking code, is compared with the moments equations. The basic moments equations, with the addition of particle decays and longitudinal emittance growth to estimate the number of particles inside of the expected rf bucket, show reasonable agreement in overall performance but fail to capture the significant beam scraping which occurs in the first few meters of the channel, resulting in a sharp drop in number of particles and in transverse emittance. Such effects are well represented by the addition of a basic beam scraping model; in addition to close agreement for the change in beam emittance and number of particles lost, the expected channel performance is closer to the ICOOL simulation. The moments equations still yield optimistic results; tracking simulations have a figure of merit which is roughly $15-20 \%$ below expected results, mainly due to longitudinal mismatch and nonlinear effects.

Here, the simple model used for beam scraping is described; this model does not allow for the possibility of a beam halo, but does reproduce well results for beams with an initial Gaussian distribution. The distribution function is assumed to be a function of transverse amplitude $A$ alone, as defined in Eq. 7. As a simple model that only adds one additional term to the transverse beam parameters, the distribution function is taken to have the form

$$
\begin{aligned}
F= & \frac{N_{G}}{\left(2 \pi m c \epsilon_{N}\right)^{2}} \exp \left(-A / 2 A_{G}\right), \quad A<\Psi A_{G} \\
& 0, \quad A>\Psi A_{G} .
\end{aligned}
$$

The extent of the scraping is characterized by a new parameter $\Psi$, so that a beam which is barely affected by apertures has large $\Psi$, and small $\Psi$ corresponds to a flattop distribution. The effect of scraping is described by two functions, $G_{1}(\Psi)=1-[1+(\Psi / 2)] \exp (-\Psi / 2)$, $G_{2}(\Psi)=1-\left[1+(\Psi / 2)+\left(\Psi^{2} / 8\right)\right] \exp (-\Psi / 2)$. The number of particles is given by $N=N_{G} G_{1}(\Psi)$, while the rms emittance of the beam is $\epsilon_{N}=A_{G} G_{2}(\Psi) / G_{1}(\Psi)$. The emittance is roughly equal to the scale "length" $A_{G}$ when $\Psi$ is large, and is $\approx \Psi A_{G} / 6$ when $\Psi$ is small.

When passing through an aperture with maximum radius $R_{\max }$, the cutoff condition is roughly described by the corresponding amplitude $A_{R} \equiv\left(P_{z} / m c\right) R_{\max }^{2} / \beta_{\perp}$. Here, it is assumed that the aperture is repeated enough times in similar cells that many betatron phases are sampled, and only the maximum radius over all betatron phases determines whether a particle is scraped. However, because there are two transverse directions, particles with amplitude above this value are not necessarily scraped, if the motion in the two transverse planes are out of phase with each other. To account for this discrepancy, the amplitude at which the beam is cut off is taken to be somewhat larger than $A_{R}$. When the beam is small, the cutoff can be taken as $A_{R}$, while for apertures much smaller than the beam, it approaches $\sqrt{3} A_{R}$. As a simple model, chosen to obtain good agreement with particle tracking simulations, in the moments equations a beam is considered to scrape against an aperture if

$$
\Psi A_{G}>A_{R} \frac{\sqrt{3}+A_{R} / 8 A_{G}}{1+A_{R} / 8 A_{G}} .
$$

Beam scraping is assumed to change the number of particles, the rms emittance, and the scraping parameter $\Psi$, but not the scale length $A_{G}$ or the weighting $N_{G}$ of the distribution function. Finally, as an approximate model for the fact that multiple scatter randomizes the beam distribution, eventually returning a scraped beam to a pure Gaussian, an equation of motion for $\Psi$ is added, having the form $\Psi^{\prime}=6 \beta_{\perp} \mathcal{S} / \epsilon_{N}$.

This full beam dynamics model has been used to analyze the cooling channel used in BNL Feasibility Study $2[8,9]$, which consists of a super-FOFO lattice where the cell structure is slightly altered down the channel to provide steadily increasing focussing at the absorber. The minimum of the beta function is originally $\approx 45 \mathrm{~cm}$, and by the end of the channel has been reduced to $20 \mathrm{~cm}$. Again, the moments equations agree well with the transverse emittance from ICOOL simulations, and yield an estimate for the figure of merit which is optimistic by roughly $15 \%$. 


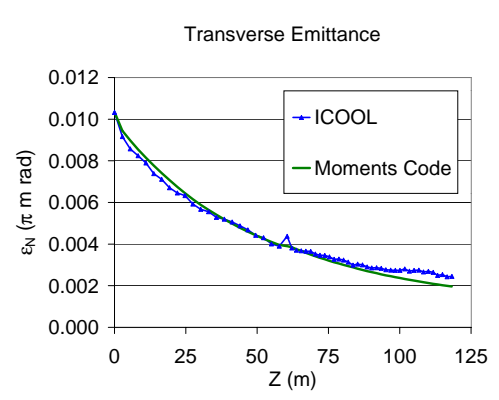

Figure 4: Transverse emittance in cooling channel used for BNL Feasibility Study 2. ICOOL simulation is compared with moments equations.

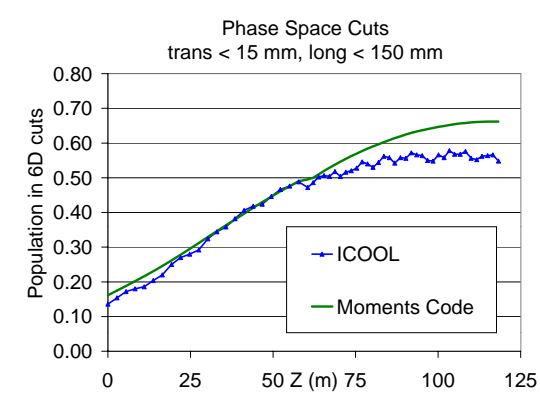

Figure 5: Evolution of figure of merit, number of particles in downstream phase space acceptance region, in cooling channel used for BNL Feasibility Study 2. ICOOL simulation is compared with moments equations.

\subsection{NONLINEAR CORRELATIONS}

Longitudinal emittance growth due to the slope of the energy loss curve in material leads to particle losses as particles move outside of the rf bucket. An important additional aspect of longitudinal dynamics is the nonlinear coupling to transverse motion. If the betatron oscillations occur faster than the synchrotron motion and beam cooling, the coupling can be expressed as terms proportional to the transverse amplitude in the equations for particle energy and time. There may be a further dependence on canonical angular momentum if the solenoid fields in the channel do not average to zero.

As particles propagate down the channel, those with high transverse amplitude will fall behind low amplitude particles of the same energy due to the increased path length. A correlation between amplitude and energy will always develop in an rf system, and mismatch in the correlation parameter will lead to large swings in energy of the particle distribution, and eventual longitudinal emittance growth or particle losses.

Here we focus on the case where $\bar{\kappa}=0$, and there is no dependence on canonical angular momentum. Then of par- ticular interest are the correlations of transverse amplitude with energy and time, defined as $C_{t}=\langle\delta t \cdot \delta A\rangle / 8 \epsilon_{N}^{2}$, $C_{E}=\langle\delta E \cdot \delta A\rangle / 8 \epsilon_{N}^{2}$. The dynamic equations for these quantities are:

$$
\begin{aligned}
C_{t}^{\prime} & =-C_{t} \frac{1}{v P} \frac{d E}{d s}-\left(C_{E}-C_{0}\right) \frac{m^{2}}{P^{3}} \\
C_{E}^{\prime} & =q \frac{\partial E_{z}}{\partial t} C_{t}-\frac{1}{v P} \frac{d E}{d s}\left(C_{E}-C_{0} m^{2} c^{4} / E^{2}\right),
\end{aligned}
$$

where $C_{0} \equiv \bar{\gamma}_{\perp} P E / 4 m c$, and $\bar{\gamma}_{\perp}$ is the average of $\gamma_{\perp}$ over several betatron oscillations. In the absence of material and thus cooling, the equilibrium or matching condition is $C_{t}=$ $0, C_{E}=C_{0}$. However, in the presence of material, the energy correlation is always pulled towards larger values, as particles are reduced in transverse amplitude but do not experience a proportional drop in energy. This problem occurs because for ionization cooling, the cooling rate is often comparable to or faster than the synchrotron period. This effect may help to explain the worse performance of cooling channels in particle tracking simulations compared to the moments equations predictions even when great care is taken to match the beam into the rf bucket.

\section{HELICAL WIGGLERS FOR EMITTANCE EXCHANGE}

Coupling between longitudinal and transverse motion must also be considered to design channels which reduce longitudinal emittance. This process is known as emittance exchange because such longitudinal cooling is done at the expense of either increased transverse emittance, or reduced transverse cooling rates. Most schemes currently being considered for emittance exchange rely on placing wedge-shaped absorbers in regions of high dispersion. The systems being considered include bent solenoids, bent lithium lenses, ring coolers, and helical wigglers. Here, helical wigglers are described and an example to reduce the energy spread of a cooled beam is given. Other methods to perform transverse cooling include stopping a beam, followed by rapid reacceleration; and rotating the matched phase space distribution out of the transverse plane, so that all components of motion experience the ionization cooling.

\subsection{HELICAL WIGGLER PROPERTIES}

A helical wiggler consists of a uniform solenoid field and a rotating dipole field. The magnetic fields are given by

$$
\begin{aligned}
& B_{x}=-2 B_{W} I_{1}^{\prime}\left(\kappa_{W} r\right) \sin \left(\kappa_{W} z\right), \\
& B_{y}=2 B_{W}\left[I_{1}\left(\kappa_{W} r\right) / \kappa_{W} r\right] \cos \left(\kappa_{W} z\right), \\
& B_{z}=B_{0}-\kappa_{W}\left(x B_{y}-y B_{x}\right),
\end{aligned}
$$

where $I_{1}$ is the modified Bessel function of first order, and $\kappa_{W}=2 \pi / L_{W}$, where $L_{W}$ is the period of rotation of the dipole field.

The stable orbit radius varies with energy, with transverse velocity that scales as $v_{\perp} \propto B_{W} / E$, and $r \propto$ 
$B_{W} /\left(\kappa_{W} E\right)$. There is a resonance when the cyclotron motion has the same period as the dipole rotation, when $P_{\text {res }}=e B_{0} L_{W} / 2 \pi$. The emittance exchange section will consist of a wiggler channel with beam momentum below resonance; the dispersion increases as the momentum approaches the resonant value. The reference orbit radius is shown in Figure 6. The wiggler parameters chosen are $L_{W}=2.5 \mathrm{~m}, B_{0}=3.2 \mathrm{~T}, B_{W}=0.2 \mathrm{~T}$. The beam momentum is $300 \mathrm{MeV} / \mathrm{c}$, which is the condition for isochronous motion in the channel; thus, the beam does not need $\mathrm{rf}$ to remain bunched.

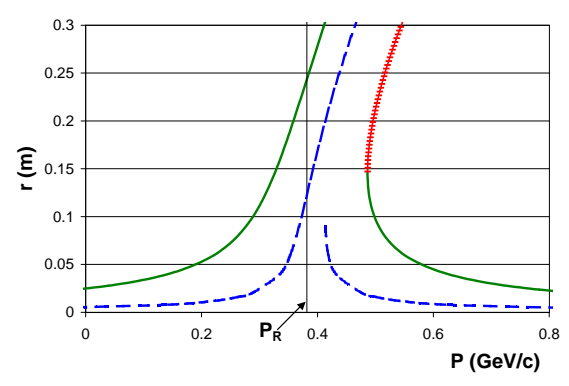

Figure 6: Radius of reference orbit as a function of beam energy. Red, hatched line corresponds to unstable orbits. Blue, dashed line corresponds to stable orbits when dipole field is reduced by factor of 5 . Resonant value of momentum is indicated.

\subsection{EMITTANCE EXCHANGE RESULTS}

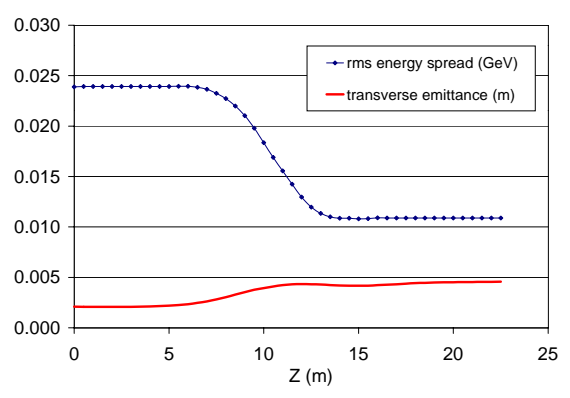

Figure 7: Variation in energy spread and transverse emittance in helical wiggler emittance exchange channel. Solenoid field is a uniform $3.2 \mathrm{~T}$ throughout.

To accomplish longitudinal cooling, wedges of beryllium are placed in regions of large beam dispersion, reducing the energy spread in the beam at the expense of increasing transverse emittance. The results are shown in Figure 7. The momentum of the beam is $300 \mathrm{MeV} / \mathrm{c}$; the other beam parameters are comparable to those at the end of the Feasibility Study 2 cooling channel, with longitudinal emittance $\approx 28 \mathrm{~mm}$, and transverse emittance $\approx 2$ $\mathrm{mm}$. The beryllium wedges reduce the energy spread by a factor of 2 . The beam remains bunched because the channel is isochronous, and all particles have forward velocities close to the maximum allowed by the channel for momenta below resonance. However, the reduced energy spread requires rebunching in order to still be matched to the previous rf system. For more details, see Ref. [10].

\section{CONCLUSIONS}

A formalism has been developed for the rapid analysis of cooling channels and as an aid in the design and understanding of such channels. With extensions to account for beam scraping and longitudinal emittance growth, calculations based on these paraxial moments equations agree well with single-particle tracking codes even of fully engineered systems, so long as sufficient care is taken to match the beam properly into the rf system. The primary nonlinear correction to these equations are the result of the variation in forward velocity with transverse amplitude; this leads to the formation of nonlinear correlations between longitudinal and transverse coordinates.

Ionization cooling is made more difficult by the fact that longitudinal emittance tends to increase during cooling. Because of the large initial longitudinal emittance of the beam, emittance exchange is desirable to reduce the energy spread in the beam, and is a necessity for a muon collider. An example using wedges in a helical wiggler channel has been shown to reduce the longitudinal emittance of a beam while preserving the bunching, if the transverse emittance of the beam is sufficiently low. Much further work is required in this area to develop a muon beam front-end which includes emittance exchange.

\section{REFERENCES}

[1] C.M. Ankenbrandt et al. (Muon Collider Collaboration), Phys. Rev. ST Accel. Beams 2, 081001 (1999).

[2] S. Geer, Phys. Rev. D57, 6989 (1998).

[3] A.N. Skrinsky and V.V. Parkhomchuk, Sov. J. Part. Nucl. 12, 223 (1981).

[4] E.D. Courant and H.S. Synder, Ann. Phys. 3, 1 (1958).

[5] K.-J. Kim and C.-X. Wang, Phys. Rev. Lett. 85(4):760-763.

[6] G. Penn and J.S. Wurtele, Phys. Rev. Lett. 85(4):764-767.

[7] R.C. Fernow, in Proceedings of the 1999 Particle Accelerator Conference, NY, p.3020.

[8] "Feasibility Study-II of a Muon-Based Neutrino Factory" BNL-52623, eds. S. Ozaki, R. Palmer, M. Zisman, J. Gallardo.

[9] R. Fernow, J. Gallardo, R. Palmer and P. Lebrun, "Ionization Cooling Scenario for a Neutrino Factory", paper FPAH079, PAC'01, Chicago, June 2001.

[10] G. Penn, J.S. Wurtele, W.M. Fawley, "Helical Channels for Longitudinal Compression of Muon Beams", paper FPAH092, PAC’01, Chicago, June 2001. 